\title{
Síndrome EPOC/asma en rehabilitación pulmonar: revisión sistemática - metaanálisis 2010 a 2015
}

\section{COPD/asthma syndrome in pulmonary rehabilitation: systematic review - meta-analysis 2010 to 2015}

\author{
Carlos-Eduardo Giraldo Ospina, MD ${ }^{1}$ Daniela González-Ramos, FTta ${ }^{2}$ \\ Carmen luisa Betancur Pulgarín, ENF ${ }^{3}$
}

\section{Resumen}

INTRODUCCIÓN: revisión sistemática de la literatura sobre estudios aleatorizados en pacientes con EPOC y asma sometidos a rehabilitación pulmonar.

MATERIALES Y MÉTODOS: se realizó una revisión sistemática en 9 bases de datos, incluida una sobre temas específicos en fisioterapia. Se filtraron los estudios a través de título y resumen, aplicando los criterios de inclusión. Los estudios seleccionados se analizaron utilizando las escalas CONSORT, Jadad y PEDro.

RESULTADOS: se filtraron 38 resultados luego de la realización de la búsqueda; de los cuales, 12 publicaciones cumplieron con estándares superiores al ser valorados por al menos 2 de las 3 escalas, siendo caracterizados como buenos o excelentes. Los estudios revisados evidenciaron una alta heterogeneidad en las intervenciones y diseños metodológicos, presentando, en múltiples ocasiones, fallos en el proceso de cegamiento tras la aleatorización.

DISCUSIÓN: no se encontraron resultados en la búsqueda fenotipo mixto-rehabilitación. Un análisis por subgrupo evidenció marcada heterogeneidad en las publicaciones que abordaron el tema EPOC-rehabilitación. No se encontraron publicaciones colombianas de impacto a nivel mundial frente al tema evaluado.

CONCLUSIÓN: de acuerdo con los datos encontrados se concluye que las intervenciones de mayor impacto en el proceso de rehabilitación pulmonar en pacientes con asma y EPOC son inciertos. El análisis por subgrupos evidencia mejoría en la funcionalidad en pacientes con EPOC luego de la aplicación de terapia domiciliaria y medicina tradicional china; no obstante, dada la naturaleza heterogénea de los datos, estos resultados se deben verificar con nuevos estudios que aborden el tema de forma integral.

Palabras clave: enfermedad pulmonar obstructiva crónica (EPOC), asma, fenotipo mixto, rehabilitación pulmonar, rehabilitación respiratoria, fenotipo mixto asma/EPOC.

\footnotetext{
Abstract

INTRODUCTION: a systematic review of the literature about randomized controlled trials in patients with overlap phenotype, which were included in pulmonary rehabilitation, is presented.

MATERIALS AND METHODS: a systematic search in nine databases, which included one about specific topics in physiotherapy, were done. Studies were filtered by title and abstract after applying inclusion criteria. Selected studies were analyzed using the CONSORT, Jadad and PEDro scales.
}

\begin{abstract}
${ }^{1}$ Médico Cirujano de la Universidad de Caldas, Médico. Cirujano especialista en Epidemiología (CC 1.053.793.981 de Barranquilla, Atlántico)

${ }^{2}$ Fisioterapeuta, Escuela Nacional del Deporte, especialista en Epidemiología (CC 1.113.634.096 de Palmira, Valle)

${ }^{3}$ Enfermera, MG Epidemiología (CC 32.311.666 de Bello, Antioquia) Autor de correspondencia Carlos Eduardo Giraldo Ospina. Carrera 52C \#94-17 Barranquilla, Atlántico. Tel.: +57 (300)797.96.10. Correo electrónico: investigamos.inmigec@gmail.com Recibido: 01/01/17. Aceptado: 01/12/17.
\end{abstract}


RESULTS: 38 results were filtrated after the systematic search. A total of 12 studies approved at least 2 of 3 scales, being characterized as good or excellent. Reviewed studies evidenced heterogeneity in the methodological design and interventions presenting failures in randomization and blinding.

DISCUSSION: no studies were found in the initial search with the mesh terms overlap phenotype and COPD. After the implementation of a subgroup analysis, was evidenced heterogeneity in the publications that centered in pulmonary rehabilitation and COPD. During the search, no Colombian high impact publications where found.

CONCLUSION: according to the found data, it is concluded that the interventions with higher impact in the pulmonary rehabilitation process in patients with asthma and COPD are unknown, the subgroup analysis evidenced improve in functionality in patients with COPD after the implementation of domiciliary therapy and Chinese traditional medicine. Nevertheless, according to the heterogeneity of the data, those results should be verified with new studies that develop in an integral way the topic.

Keywords: chronic obstructive pulmonary disease (COPD), asthma, overlap phenotype, pulmonary rehabilitation, respiratory rehabilitation.

\section{Introducción}

La rehabilitación pulmonar es una disciplina emergente que ha tomado fuerza en los últimos años debido al manejo interdisciplinario que se realiza en los pacientes con patologías crónicas y su enfoque hacia la mejoría en la calidad de vida (1). Una de las patologías crónicas con mayor impacto a nivel mundial es la enfermedad pulmonar obstructiva crónica (EPOC), la cual presenta un marcado patrón de ascenso en los últimos años. Según lo demostrado por los resultados de distintas estrategias, como PLATINO, BOLD y GOLD, para el año 2020 se espera que la EPOC sea la tercera causa de mortalidad, siendo a 2015 la cuarta causa de muerte a nivel mundial (2-7).

Se han postulado teorías respecto al comportamiento de la EPOC como un síndrome que incluye múltiples patologías que interactúan de forma continua $(8,9)$. Ante este hecho, se ha propuesto que el asma y EPOC son una única manifestación de enfermedades con patrón obstructivo, y que la presencia de asma en la infancia predispone al desarrollo de EPOC en la adultez y senectud. Los nuevos hallazgos en el comportamiento de la EPOC ponen en entredicho la naturaleza unitaria de la enfermedad $(10,11)$. Ante dichos planteamientos, en 2010, la Sociedad Española de Neumología y Cirugía Torácica (SEPAR) introdujo el concepto de fenotipos en la EPOC, incluido el fenotipo mixto u overlap, donde existe una obstrucción al flujo espiratorio parcialmente reversible (12).
Los siguientes fenotipos se consideran como los principales de la EPOC: fenotipo agudizador, que se define como pacientes que presentaron más de 2 exacerbaciones de su patología base en el último año, cada 1 con intervalo de al menos 4 semanas, si presentaron tratamiento, o 6 semanas si no se tuvo manejo; el fenotipo mixto u overlap que corresponde a los pacientes con obstrucción no completamente reversible al flujo aéreo, acompañado de síntomas o signos de una reversibilidad aumentada de la obstrucción; y el fenotipo enfisematoso, en el cual el hallazgo predominante es intolerancia al ejercicio o disnea, como síntomas predominantes, que se acompañan con signos radiológicos de hiperinsuflación. Se han encontrado asociaciones descritas entre la presencia del fenotipo enfisematoso y pacientes con índice de masa corporal (IMC) bajo (13-20).

Con el presente estudio se busca realizar una revisión sistemática y metaanálisis sobre los resultados de estudios experimentales aleatorizados en pacientes con diagnóstico de EPOC y asma sometidos a rehabilitación pulmonar y valorados con la caminata de los 6 minutos entre los años 2010-2015 para identificar el estado actual de la rehabilitación pulmonar en EPOC-asma, partiendo de la hipótesis que existe heterogeneidad en los estudios aleatorizados encontrados en las bases de datos internacionales sobre rehabilitación pulmonar en pacientes adultos con EPOC-asma. 


\section{Materiales y métodos}

Se presenta un estudio integrativo, de corte trasversal y retrospectivo, para el cual se realizó un protocolo de búsqueda de la literatura, en el que 2 observadores de forma independiente filtraron la información de 9 bases de datos: PubMed, Scopus, ScienceDirect, EBSCO, Ovid, BIREME y SciELO, incluido una base de datos sobre temas específicos de fisioterapia denominada PEDro.

\section{Criterios de inclusión}

- Artículos experimentales aleatorizados en pacientes adultos con fenotipo mixto o EPOC y asma sometidos a rehabilitación pulmonar.

- Artículos publicados en las bases datos: PubMed, Scopus, ScienceDirect, EBSCO, Ovid, BIREME, SciELO y PEDro.

- Artículos de cualquier nacionalidad publicados en idioma inglés o español.

- Artículos ubicados con los siguientes descriptores:

- DeCS: enfermedad pulmonar obstructiva crónica o EPOC, asma, rehabilitación pulmonar o rehabilitación respiratoria.

- Mesh: pulmonary disease, chronic obstructive, or COPD, asthma, pulmonary rehabilitation.

\section{Criterios de exclusión}

- Artículos que no cumplan con el desenlace: funcionalidad evaluada, como caminata de los 6 minutos.

- Artículos que no cumplan con suficiente validez interna según la estrategia CONSORT, Jadad y PEDro.

- Artículos que, cumpliendo con los criterios de inclusión, no resulten pertinentes respecto al objetivo general.

Una vez filtrados los artículos se procedió a evaluar cada publicación a través de las escalas CONSORT, Jadad y PEDro; cada autor valoró las publicaciones seleccionadas por separado (21-23). Al final se determinó la validez de la evaluación a través de un análisis estadístico de correlación y concordancia. Se utilizó estadística descriptiva e inferencial para el desarrollo y síntesis de los resultados. Las variables a analizar fueron las determinadas por las estrategias CONSORT, Jadad y PEDro.

\section{Resultados}

Se realizó una búsqueda sistemática con los términos fenotipo mixto asma-EPOC y rehabilitación para publicaciones en español y overlap phenotype or (chronic obstructive pulmonary disease and asthma) and rehabilitation para publicaciones en inglés. En las bases de datos seleccionadas, donde se evidenció que no existe información en conjunto para los dos términos (fenotipo mixto-rehabilitación), se replanteó la búsqueda de la siguiente manera (Figura 1): se realizó una búsqueda sistemática de la literatura en las 9 bases de datos citadas, encontrando 56 publicaciones seleccionadas, de las cuales 38 publicaciones, que correspondieron al $67,9 \%$ del total inicial, fueron analizadas. Se eliminaron 17 publicaciones por no corresponder a estudios experimentales, 2 textos no se encontraban de forma completa y 4 publicaciones correspondían a protocolos sin resultados; se excluyó 1 publicación por ser un tema no relevante. De las 38 publicaciones analizadas, 12 artículos presentaron valoraciones de consistencia interna altas: $50 \%$ con resultados altos en las 3 escalas, y las publicaciones restantes con resultados altos en 2 de las 3 escalas valoradas.

Cada publicación fue valorada con la escala CONSORT, Jadad y PEDro por 2 evaluadores de manera independiente. Se verificó la concordancia de ambas valoraciones a través de la prueba kappa de Cohen para las clasificaciones, y el coeficiente de correlación intraclase de Lin para las puntuaciones totales. Los detalles de dichos análisis se encuentran disponibles en el documento extenso.

Se encontró una relación estadísticamente significativa entre los evaluadores al comparar el desempeño con la prueba Jadad, no así con los resultados en la escala CONSORT y PEDro. Se halló una concordancia del 52,6\% (kappa: 0,22) para las valoraciones con la escala CONSORT; en el caso de la escala PEDro fue del 68,42\% (kappa: 0,30); y para las valoraciones de 


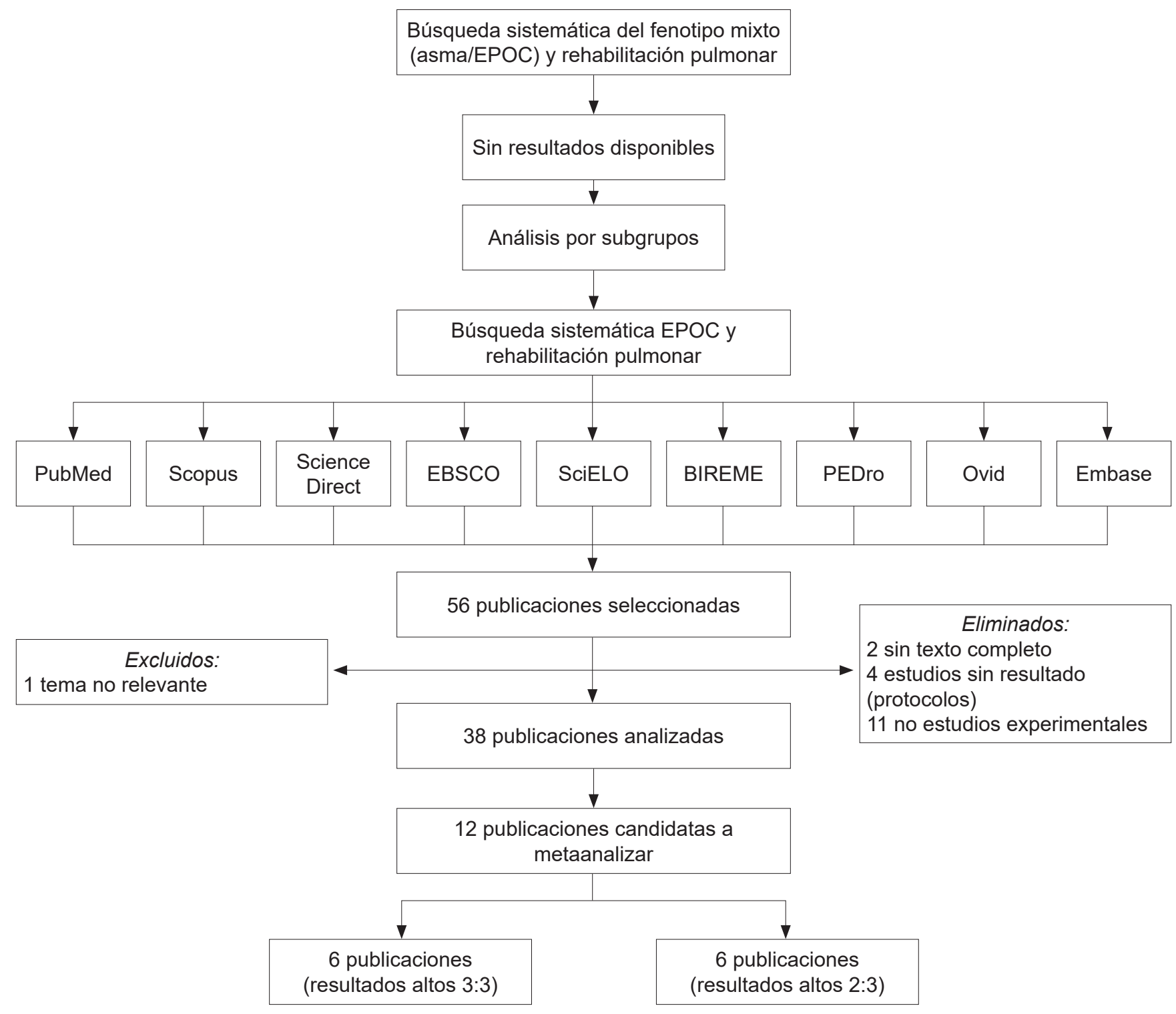

Figura 1. Árbol de decisiones.

la escala Jadad se valoró una concordancia del 92,11\% (kappa: 0,86). Para las tres valoraciones se contó con un valor $p$ estadísticamente significativo (Tabla 1).

De los 38 artículos que contaron con al menos 2 de 3 valoraciones con calificaciones superiores se seleccionaron 12. Para ello se utilizaron los siguientes puntos de corte: para la escala Jadad superior a 6 puntos, excelente, y entre 4 a 5 puntos, bueno; para la escala PEDro superior a 6 puntos, bueno; para la escala CONSORT entre 26 a 30 puntos, bueno, y mayor de 31 puntos, excelente (Tabla 1).
Las 12 publicaciones seleccionadas se dividieron en 2 grupos de acuerdo con los resultados del análisis de consistencia interna arrojado por las 3 escalas de valoración. En ambos subgrupos se encontró un patrón de distribución de sesgos similar. Uno y otro contaron con un adecuado proceso de aleatorización y ocultamiento, al igual que un desarrollo apropiado en la exposición de resultados. En el reporte de citaciones se sostuvo un alto riesgo de sesgo en el ocultamiento de los desenlaces pese a la consistencia interna de las 12 publicaciones (Figura 2) (24-35). 
Tabla 1. Calificación de los artículos según las escalas con las que se revisaron

\begin{tabular}{|cccccc|}
\hline Escala & Concordancia & Índice kappa & Valor P & Puntos de corte & Concepto \\
Jadad & $92,11 \%$ & 0,86 & 0,00 & $6-7$ & Excelente \\
& & & & $4-5$ & Bueno \\
& & & & $0-3$ & Débil \\
PEDro & $68,42 \%$ & 0,30 & 0,02 & $6-11$ & Bueno \\
& & & & $0-5$ & Débil \\
\hline CONSORT & \multirow{2}{*}{$52,6 \%$} & 0,22 & 0,01 & $31-37$ & Excelente \\
& & & & $26-30$ & Bueno \\
& & & & $0-15-25$ & Regular \\
& & & & Débil \\
\hline
\end{tabular}

Análisis de sesgos

Publicaciones con muy alta consistencia interna

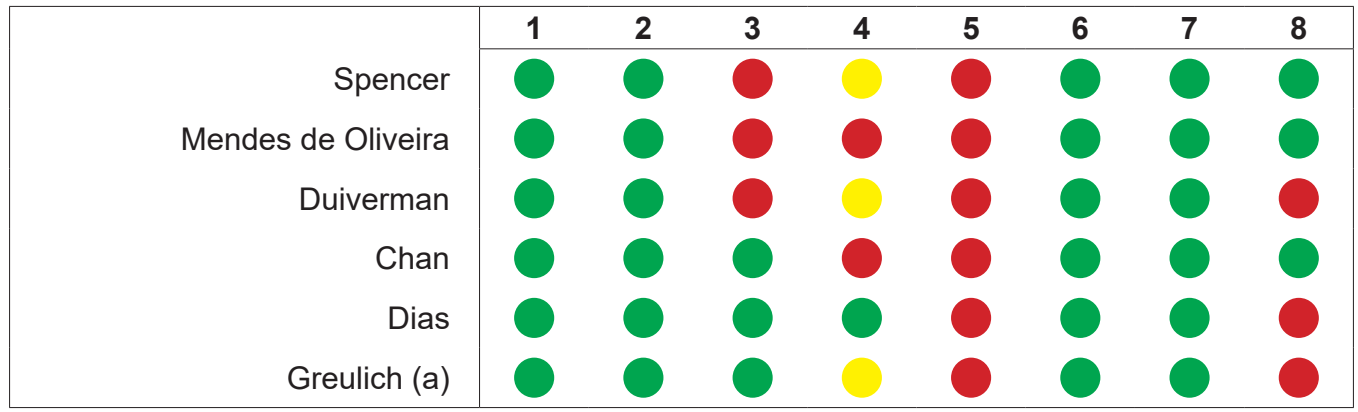

Publicaciones con alta consistencia interna

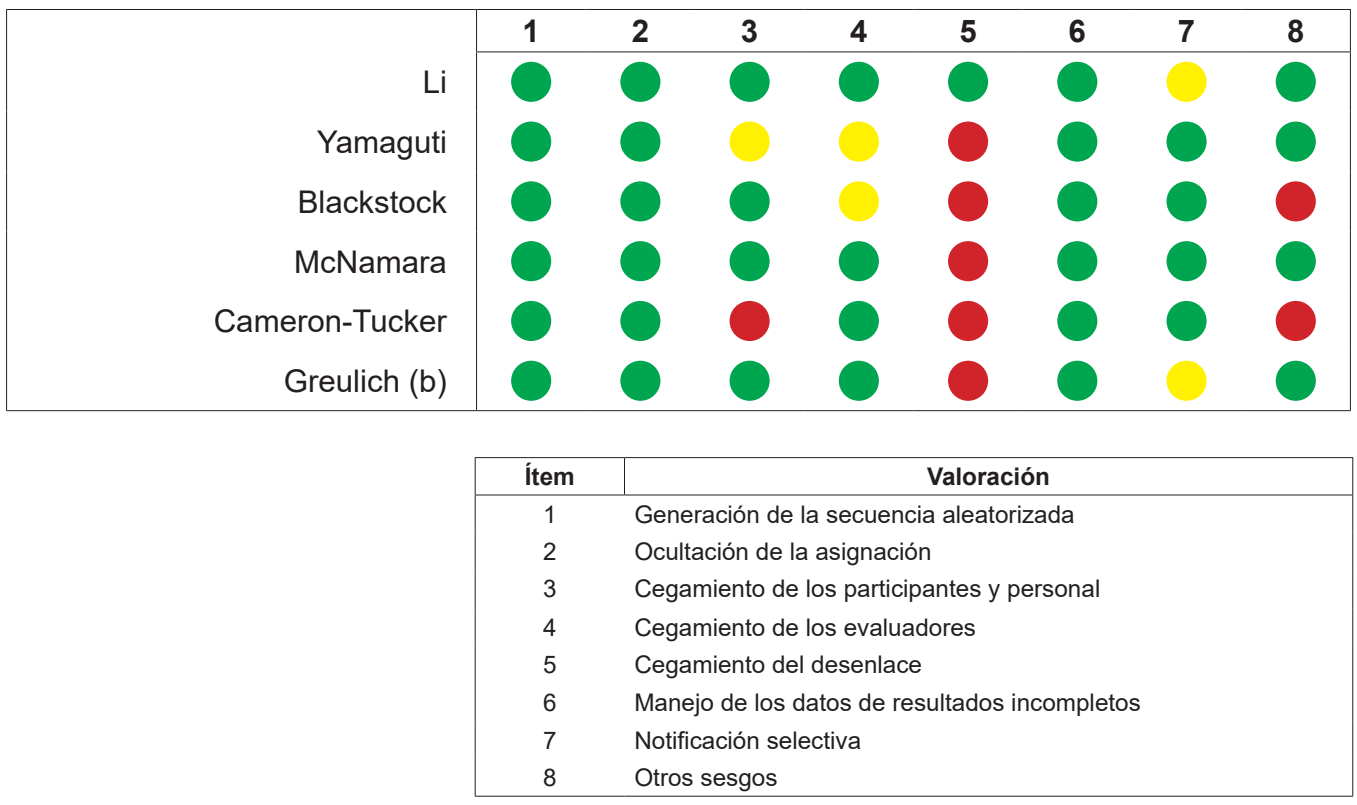

Figura 2. Análisis de sesgos de publicaciones con alta y muy alta consistencia interna. 
En cuanto al grupo de publicaciones con muy alta consistencia interna, se encontró que $50 \%$ de las publicaciones contó con alto riesgo de sesgo en el proceso de cegamiento de los participantes y personal. El 100\% refirió fallos en el cegamiento del desenlace, y $50 \%$ presentó alto riesgo de sesgos en el ítem que valoró otros sesgos. Frente al cegamiento de solo evaluadores, una publicación contó con bajo riesgo de sesgo. Mientras que para las publicaciones con alta consistencia interna se encontró menor proporción de riesgos de sesgo en el cegamiento de participantes y personal, contando con un caso de alto riesgo de sesgo y uno de riesgo poco claro. El comportamiento para el cegamiento de los evaluadores fue superior al grupo de muy alta consistencia interna, reportando solo $33,3 \%$ de los estudios con riesgo poco claro y ausencia de reportes con alto riesgo de sesgo. El comportamiento para el cegamiento del desenlace fue similar al grupo de muy alto riesgo de sesgo, contando solo con un estudio con bajo riesgo de sesgo.

Dada la heterogeneidad de las publicaciones analizadas se utilizó el modelo de efectos aleatorios bajo la prueba de DerSimonian y Laird, con un intervalo de confianza (IC) del 95\%. Cada publicación se disgregó según los tiempos de intervención, lo que implicó multiplicar las intervenciones en función de los tiempos de seguimiento. Las valoraciones generales corresponden a la primera publicación en todos los casos de duplicación. Cada proceso se revisó por el método homólogo de análisis de efectos fijos.

El total de intervenciones realizadas evidenció una tendencia a favor de los procesos de rehabilitación pulmonar respecto al placebo, mostrando mejoría en relación con los resultados en la caminata de los 6 minutos. No obstante, con un nivel de dispersión en las publicaciones que dificulta su interpretación, el análisis de las intervenciones en función de sus factores individuales mostró un patrón de mayor uniformidad.

En cuanto a los resultados en las publicaciones, se encontró un marcado nivel de heterogeneidad. Para las 12 intervenciones se halló un valor Tau de 359,5 $(p=0,001)$ $Q=100 \%$. En el modelo de efectos fijos se descubrió un valor Tau de $114576780(p=0,001) Q=100 \%$, evidenciando una marcada discordancia. En el modelo de efectos aleatorios, los valores de heterogeneidad para las intervenciones disgregadas fueron de 201,2 $(p=0,001)$ $Q=100 \%$; mientras que, para el modelo de efectos fijos, el valor Tau correspondió al 256550922,5 $(p=0,001)$ $Q=100 \%$, soportando los valores de heterogeneidad antes reportados. Los intervalos de confianza de las 12 intervenciones se exponen en la (Figura 3).

\section{Análisis por subgrupos}

Las intervenciones se valoraron de acuerdo con el tipo, tiempo de intervención y de seguimiento, y tamaño de la muestra, encontrando los siguientes resultados.

\section{Resultados por tiempo de intervención}

El análisis según el tiempo de intervención y seguimiento no mostró valores $p$ estadísticamente significativos para las intervenciones por separado. Se encontró persistencia de la heterogeneidad en las intervenciones contrastadas; no obstante, las intervenciones menores o iguales de 12 semanas mostraron un comportamiento sugerente de mejora respecto a intervenciones en tiempos superiores. No se encontró un patrón de decisión claro para intervenciones superiores a las 48 semanas (Figura 4).

\section{Resultados por tamaño de muestra}

Se encontró que los estudios correspondientes a Chan y colaboradores, al igual que Yamaguti y colaboradores, presentaron tamaños de muestra que desplazaron las intervenciones restantes $(27,31)$. Se hallaron tamaños de muestra con bajos números, lo cuales aumentaron los intervalos de confianza de los distintos estudios, así como incremento en el arrastre dado la multiplicación de las intervenciones en función del tiempo. Para limitar este efecto se procedió a analizar cada intervención por separado.

\section{Resultados por tipo de intervención}

En las 12 publicaciones analizadas (Tabla 2) se encontraron, como intervenciones significativas, el uso de medicina tradicional china, modelos de entrenamiento muscular, seguimiento de los pacientes sometidos a procesos de rehabilitación y asociación de ventilación mecánica no invasiva. 
Conjunto de estudios

Spencer y colaboradores: seguimiento de 12 semanas

+ Mendes de Oliveira y colaboradores: 12 semanas de rehabilitación pulmonar domiciliaria

+ Duiverman y colaboradores: 12 semanas de rehabilitación pulmonar y ventilación mecánica no invasiva

+ Chan y colaboradores: 6 semanas de rehabilitación pulmonar tradicional

+ Dias y colaboradores: 8 semanas de rehabilitación pulmonar domiciliaria

+ Greulich y colaboradores: 12 semanas de tradicional china

+ Yamaguti y colaboradores: 4 semanas de rehabilitación pulmonar y entrenamiento diafragmático

+ Blackstock y colaboradores: 48 semanas de rehabilitación pulmonar individual

+ McNamara y colaboradores: 8 semanas de rehabilitación pulmonar con hidroterapia

+ Cameron-Tucker y colaboradores: 6 semanas de rehabilitación pulmonar

+ Greulich y colaboradores: abierto en tiempo.

Entrenamiento vibratorio tratamiento individualizado $v s$. grupal

+ Li y colaboradores: 12 semanas. Medicina

Estimación acumulada

Diferencia de media estandarizada

\begin{tabular}{rrr}
5,902 & $(4,592 ;$ & $7,211)$ \\
$-31,445$ & $(-105,077 ;$ & $42,187)$ \\
$-30,572$ & $(-67,215 ;$ & $6,072)$ \\
$-102,481$ & $(-151,709 ;$ & $-53,252)$ \\
$-34,068$ & $(-52,126 ;$ & $-16,009)$ \\
$-33,600$ & $(-50,261 ;$ & $-16,938)$ \\
$-28,096$ & $(-40,864 ;$ & $-15,328)$ \\
$-31,312$ & $(-44,390 ;$ & $-18,234)$ \\
$-24,865$ & $(-39,377 ;$ & $-10,352)$ \\
$-26,576$ & $(-40,311 ;$ & $-12,840)$ \\
$-17,458$ & $(-30,992 ;$ & $-3,924)$ \\
$-14,365$ & $(-28,187 ;$ & $-0,544)$ \\
\hline
\end{tabular}

Estimación acumulada (IC 95\%)

\section{Estudios}

Spencer y colaboradores: seguimiento de 12 semanas (2010)

Mendes de Oliveira y colaboradores: 12 semanas de rehabilitación pulmonar domiciliaria (2010)

Duiverman y colaboradores: 12 semanas de rehabilitación pulmonar y ventilación mecánica no invasiva (2011)

Chan y colaboradores: 6 semanas de rehabilitación pulmonar tradicional (2011)

Dias y colaboradores: 8 semanas de rehabilitación pulmonar domiciliaria (2013)

Greulich y colaboradores: 12 semanas de tratamiento individualizado vs. grupal (2014)

Li y colaboradores: 12 semanas. Medicina tradicional china (2012)

Yamaguti y colaboradores: 4 semanas de rehabilitación pulmonar y entrenamiento diafragmático (2012)

Blackstock y colaboradores: 48 semanas de rehabilitación pulmonar individual (2013)

McNamara y colaboradores: 8 semanas de rehabilitación pulmonar con hidroterapia (2014)

Cameron-Tucker y colaboradores: 6 semanas de rehabilitación pulmonar (2014)

Greulich y colaboradores: abierto en tiempo.

Entrenamiento vibratorio (2014)

$\begin{array}{rrr}5,902 & (4,592 ; & 7,211) \\ -69,236 & (-80,633 ; & -57,838) \\ -29,581 & (-35,085 ; & -24,077) \\ -2088,132 & (-2337,204 ; & -1839,060) \\ -5,120 & (-6,814 ; & -3,426) \\ -28,794 & (-33,928 ; & -23,660) \\ -15,517 & (-16,767 ; & -14,268) \\ & & \\ -875,639 & (-1097,203 ; & -654,075) \\ & & \\ 36,895 & (33,757 ; & 40,034) \\ -37,333 & (-45,070 ; & -29,595) \\ 66,055 & (56,057 ; & 76,052) \\ 1377,520 & (1104,789 ; & 1650,251) \\ -14,365 & (-28,187 ; & -0,544)\end{array}$

Total $\left(I^{\wedge} 2=99 \% ; p<0,001\right)$

$-0,544)$

Diferencia de media estandarizada

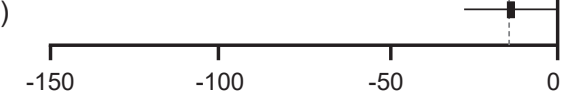

Figura 3. Ajuste de intervenciones por intervalos de confianza. 
Estudios

Spencer y colaboradores: seguimiento de 12 semanas

Mendes de Oliveira y colaboradores: 12 semanas de rehabilitación pulmonar domiciliaria

Mendes de Oliveira y colaboradores: 12 semanas de rehabilitación pulmonar convencional

Duiverman y colaboradores: 12 semanas de rehabilitación pulmonar y ventilación mecánica no invasiva

Chan y colaboradores: 12 semanas de rehabilitación pulmonar tradicional

Chan y colaboradores: 12 semanas de rehabilitación pulmonar y medicina tradicional china

Greulich y colaboradores: 12 semanas de tratamiento individualizado vs. grupal

Li y colaboradores: 12 semanas de medicina tradicional china

Subgrupo $12\left(\left.\right|^{\wedge} 2=100 \% ; p=0,000\right)$

Spencer y colaboradores: seguimiento de 24 semanas

Duiverman y colaboradores: 24 semanas de rehabilitación pulmonar

y ventilación mecánica no invasiva

Subgrupo $24\left(I^{\wedge} 2=100 \% ; p=0,000\right)$

Spencer y colabores: seguimiento de 48 semanas

Duiverman y colabores: 48 semanas de rehabilitación pulmonar y

ventilación mecánica no invasiva

Blackstock y colabores: 48 semanas de rehabilitación pulmonar

individual

Blackstock y colabores: 48 semanas de rehabilitación pulmonar

grupal

Blackstock y colabores: 48 semanas de rehabilitación pulmonar grupal y educación

Subgrupo $48\left(I^{\wedge} 2=100 \% ; p=0,000\right)$

Duiverman y colaboradores: 96 semanas de rehabilitación pulmonar y ventilación mecánica no invasiva

Subgrupo 96 ( $\left.{ }^{\wedge} 2=\mathrm{NA} ; p=\mathrm{NA}\right)$

Chan y colaboradores: 6 semanas de rehabilitación pulmonar tradicional

Chan y colaboradores: 6 semanas de rehabilitación pulmonar y medicina tradicional china

Cameron-Tucker y colaboradores: 6 semanas de rehabilitación pulmonar

Subgrupo $6\left(I^{\wedge} 2=100 \% ; p=0,000\right)$

Dias y colaboradores: 8 semanas de rehabilitación pulmonar domiciliaria

McNamara y colaboradores: 8 semanas de rehabilitación pulmonar con hidroterapia

McNamara y colaboradores: 8 semanas de rehabilitación pulmonar sin hidroterapia

Subgrupo $8\left(I^{\wedge} 2=100 \% ; p=0,000\right)$

Li y colaboradores: 18 semanas de medicina tradicional china Subgrupo $18\left(I^{\wedge} 2=\mathrm{NA} ; p=\mathrm{NA}\right)$

Li y colaboradores: 72 semanas de medicina tradicional china Subgrupo $72\left(I^{\wedge} 2=N A ; p=N A\right)$

Yamaguti y colaboradores: 4 semanas de rehabilitación pulmonar y entrenamiento diafragmático

Subgrupo 4 (I^2=NA; $p=N A)$

Greulich y colaboradores: abierto en tiempo. Entrenamiento vibratorio

Subgrupo $0\left(I^{\wedge} 2=N A ; p=N A\right)$

Total $\left(\left.\right|^{\wedge} 2=100 \% ; p=0,000\right.$
Estimación (IC 95\%)

\begin{tabular}{|c|c|c|}
\hline 3,000 & $(2,717$ & $3,283)$ \\
\hline$-35,000$ & $(-35,237$ & $-34,763)$ \\
\hline 15,000 & $(14,791 ;$ & $15,209)$ \\
\hline$-15,000$ & $(-15,265$ & $-14,735)$ \\
\hline$-23,000$ & $(-23,003$ & $-22,997)$ \\
\hline 4,000 & $(3,997$; & $4,003)$ \\
\hline$-3,500$ & $(-3,560$ & $-3,440)$ \\
\hline$-7,000$ & $(-7,101$ & $-6,899)$ \\
\hline$-7,687$ & $(-20,876$ & $5,501)$ \\
\hline 4,500 & $(4,217$ & $4,783)$ \\
\hline$-25,000$ & $(-25,265$ & $-24,735)$ \\
\hline$-10,250$ & $(-39,160$ & $18,659)$ \\
\hline 9,500 & $(9,217$ & $9,783)$ \\
\hline$-35,000$ & $(-35,265$ & $-34,735)$ \\
\hline 18,500 & $(18,380 ;$ & $18,620)$ \\
\hline 19,000 & $(18,880$ & $19,120)$ \\
\hline 18,000 & $(17,880$ & $18,120)$ \\
\hline 6,000 & $(-7,149$ & $19,150)$ \\
\hline$-65,000$ & $(-65,265$ & $-64,735)$ \\
\hline$-65,000$ & $(-65,265$ & $-64,735)$ \\
\hline$-21,000$ & $(-21,003$ & $-20,997)$ \\
\hline$-2,000$ & $(-2,003 ;$ & $-1,997)$ \\
\hline 2,000 & $(1,987$; & $2,013)$ \\
\hline$-7,000$ & $(-21,973$; & $7,973)$ \\
\hline$-34,000$ & $(-39,276$ & $-28,724)$ \\
\hline$-19,000$ & $(-19,310$ & $-18,690)$ \\
\hline 34,000 & $(33,690$; & $34,310)$ \\
\hline$-6,286$ & $(-48,710$ & $36,138)$ \\
\hline$-12,500$ & $(-12,601$ & $-12,399)$ \\
\hline$-12,500$ & $(-12,601$ & $-12,399)$ \\
\hline$-17,500$ & $(-17,601$ & $-17,399)$ \\
\hline$-17,500$ & $(-17,601$ & $-17,399)$ \\
\hline$-9,000$ & $(-9,007$; & $-8,993)$ \\
\hline$-9,000$ & $(-9,007$; & $-8,993)$ \\
\hline 14,000 & $(13,994 ;$ & $14,006)$ \\
\hline 14,000 & $(13,994 ;$ & $14,006)$ \\
\hline$-6,964$ & $(-12,421$ & $-1,507)$ \\
\hline
\end{tabular}
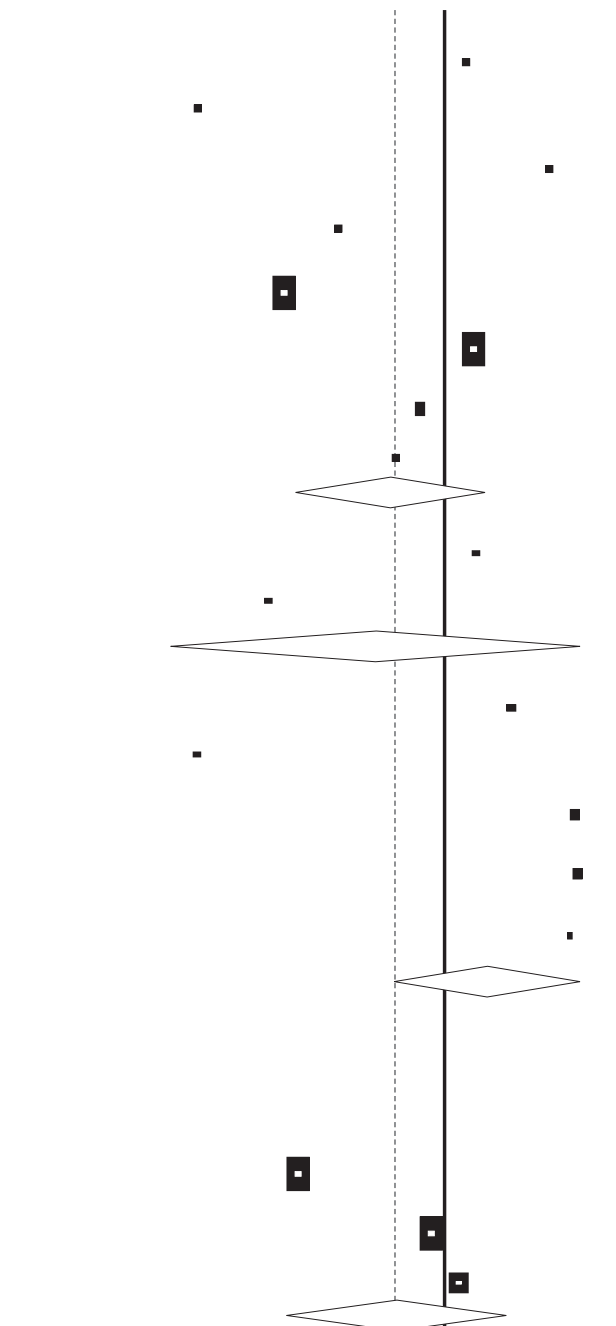
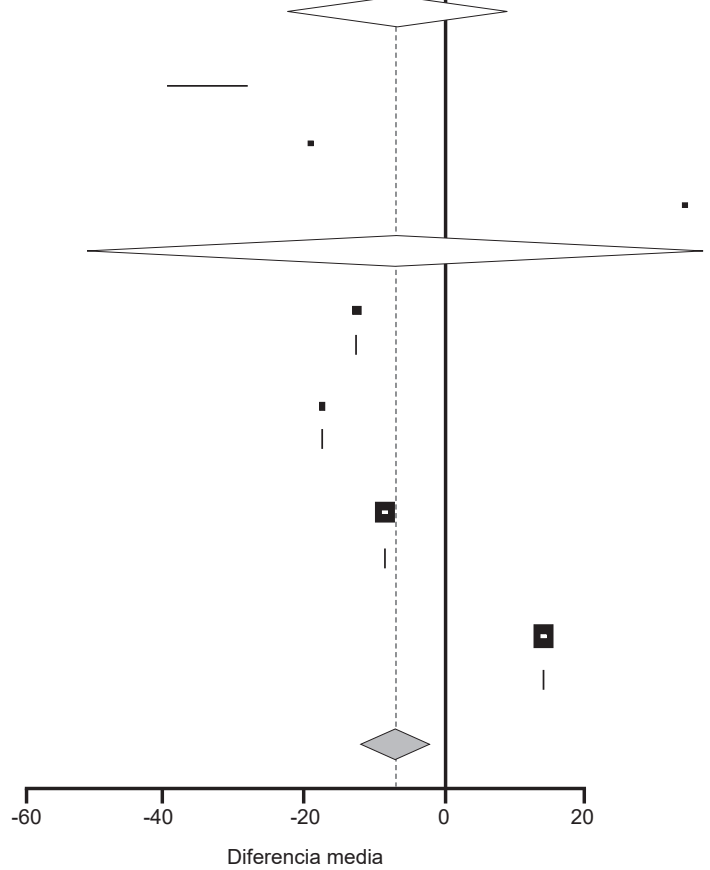

Figura 4. Análisis según tiempo de intervención.

14 Revista Colombiana de Neumología Vol. 29 N. ${ }^{\circ} 2$ | 2017 
Frente a la intervención basada en medicina tradicional china se analizó el estudio de Chan y colaboradores, al igual que la de Li y colaboradores, encontrando mejoría en la caminata de los 6 minutos respecto a la no intervención $(27,30)$. No obstante, los resultados fueron desplazados por el tamaño de muestra de la intervención realizada por Chan. Las intervenciones donde se valoró el seguimiento de los pacientes evidenciaron resultados discordantes, no hubo significancia estadística de acuerdo con las publicaciones analizadas frente al entrenamiento en rehabilitación.

Con respecto a la hidroterapia en rehabilitación, la intervención propuesta por McNamara y colaboradores (33) contó con un mayor peso tanto en el modelo de efectos aleatorios como en el de efectos fijos $(49,99 \%$ y $99,84 \%$, respectivamente). Duiverman y colaboradores aportaron la intervención bajo ventilación mecánica no invasiva. Al analizar sus respectivos subgrupos se encontraron resultados no concluyentes que se explican en función de los tiempos de seguimiento en cada intervención; no se halló mejoría significativa de acuerdo con los datos provistos (Figura 5).

Cada una de las intervenciones ajustadas por tamaño de muestra se descompuso, según el tiempo de intervención y tamaño de la muestra, por cada momento de la valoración, arrojando un patrón de dispersión directamente proporcional al tiempo de duración de las intervenciones; hecho que, a su vez, se generó por la disminución de participantes en función del tiempo de intervención.

\section{Discusión}

Al realizar la revisión sistemática de la literatura sobre los resultados de estudios experimentales aleatorizados de paciente con fenotipo mixto o EPOC y asma sometidos a rehabilitación pulmonar y valorados con la caminata de los 6 minutos entre los años 2010 a 2015, no se encontraron publicaciones que cumplieran con los requerimientos delimitados en los criterios de inclusión, de acuerdo con los datos suministrados por el metabuscador Scopus. En los últimos 5 años, el total de publicaciones referentes al fenotipo mixto fue de 71 resultados, con 7 publicaciones en 2010 y 18 artículos en 2014 y 2015. De estos, el 54,9\% correspondió a ar- tículos de corte trasversal, principalmente, y el 39,4\% a revisiones narrativas $(11,17)$.

Respecto a la progresión de los pacientes con fenotipo mixto, un estudio de cohorte de Fu y colaboradores evaluó el comportamiento de los pacientes con EPOC, asma y fenotipo mixto, tomando como desenlace múltiples escalas de predicción de mortalidad, como la caminata de los 6 minutos (36). Estos autores encontraron que los pacientes con diagnóstico de EPOC presentaban un deterioro más marcado al compararlos con aquellos con fenotipo mixto, elementos que fueron representativos en la prueba de caminata de los 6 minutos. No se encontraron datos que evaluaran la rehabilitación. Los autores concluyeron que existe una necesidad de nuevos estudios (12).

En 2015, Postma y colaboradores publicaron una revisión narrativa sobre el síndrome del asma-EPOC, resaltando la importancia del problema actualmente enunciado, y evidenciando la creciente cantidad de publicaciones indexadas sobre el tema (37). Estos autores abordaron el fenotipo mixto desde los comportamientos espirométricos y el patrón de hiperreactividad bronquial, hallazgos concordantes con lo expuesto por Fu y colaboradores (36).

Una vez realizado el análisis por subgrupos, se encontró una amplia cantidad de publicaciones que abordan el tema EPOC-rehabilitación pulmonar. Se realizó una revisión de la literatura en el buscador PubMed, donde se evidenciaron 28 metaanálisis relacionados con el tema en los últimos 5 años, y 46 revisiones sistemáticas en el mismo período. Con respecto al papel de la rehabilitación pulmonar en EPOC, se encontró un metaanálisis del grupo Health Quality Ontario donde posicionaron la rehabilitación pulmonar como elemento clave en el tratamiento de la EPOC (38). Este hallazgo fue concordante con los datos de McCarthy y colaboradores en una revisión sistemática y metaanálisis con la colaboración Cochrane (39).

De acuerdo con los resultados obtenidos, se resaltó el papel de 2 intervenciones: en la primera se encontró un metaanálisis publicado en el año 2014, donde se valoraron 11 publicaciones, y se concluyó que la terapia tai chi puede usarse de forma segura en pacien- 


\section{Estudios}

Spencer y colaboradores: seguimiento de 12 semanas

Spencer y colaboradores: seguimiento de 24 semanas

Spencer y colaboradores: seguimiento de 48 semanas

Subgrupo $48\left(I^{\wedge} 2=100 \% ; p=0,000\right)$

Mendes de Oliveira y colaboradores: 12 semanas de rehabilitación pulmonar domiciliaria

Mendes de Oliveira y colaboradores: 12 semanas de rehabilitación pulmonar convencional

Subgrupo $117\left(\left.\right|^{\wedge} 2=100 \% ; p=0,000\right)$

Duiverman y colaboradores: 12 semanas de rehabilitación pulmonar y ventilación mecánica no invasiva

Duiverman y colaboradores: 24 semanas de rehabilitación pulmonar y ventilación mecánica no invasiva

Duiverman y colaboradores: 48 semanas de rehabilitación pulmonar y ventilación mecánica no invasiva

Duiverman y colaboradores: 96 semanas de rehabilitación pulmonar y ventilación mecánica no invasiva

Subgrupo $56\left(I^{\wedge} 2=100 \% ; p=0,000\right)$

Chan y colaboradores: 6 semanas de rehabilitación pulmonar tradicional

Chan y colaboradores: 12 semanas de rehabilitación pulmonar tradicional

Chan y colaboradores: 6 semanas de rehabilitación pulmonar y medicina tradicional china

Chan y colaboradores: 12 semanas de rehabilitación pulmonar y

medicina tradicional china

Subgrupo $206\left(I^{\wedge} 2=100 \% ; p=0,000\right)$

Dias y colaboradores: 8 semanas de rehabilitación pulmonar domiciliaria

Subgrupo $23\left(I^{\wedge} 2=N A ; p=N A\right)$

Greulich y colaboradores: 12 semanas de tratamiento individualizado vs. grupal

Subgrupo $23\left(I^{\wedge} 2=\mathrm{NA} ; p=\mathrm{NA}\right)$

Li y colaboradores: 12 semanas de medicina tradicional china

Li y colaboradores: 18 semanas de medicina tradicional china

Li y colaboradores: 72 semanas de medicina tradicional china

Subgrupo $306\left(I^{\wedge} 2=100 \% ; p=0,000\right)$

Yamaguti y colaboradores: 4 semanas de rehabilitación pulmonar y entrenamiento diafragmático

Subgrupo 30 ( $\left.\left.\right|^{\wedge} 2=\mathrm{NA} ; p=\mathrm{NA}\right)$

Blackstock y colabores: 48 semanas de rehabilitación pulmonar individual

Blackstock y colabores: 48 semanas de rehabilitación pulmonar grupal

Blackstock y colabores: 48 semanas de rehabilitación pulmonar grupal y educación

Subgrupo $267\left(I^{\wedge} 2=98 \% ; p=0,000\right)$

McNamara y colaboradores: 8 semanas de rehabilitación pulmonar con hidroterapia

McNamara y colaboradores: 8 semanas de rehabilitación pulmonar sin hidroterapia

Subgrupo $8\left(I^{\wedge} 2=100 \% ; p=0,000\right)$

Cameron-Tucker y colaboradores: 6 semanas de rehabilitación pulmonar

Subgrupo $84\left(I^{\wedge} 2=N A ; p=N A\right)$

Greulich y colaboradores: abierto en tiempo. Entrenamiento vibratorio Subgrupo $49\left(I^{\wedge} 2=N A ; p=N A\right)$

Total $\left(I^{\wedge} 2=100 \% ; p=0,000\right)$

\begin{tabular}{|c|c|c|}
\hline \multicolumn{3}{|c|}{ Estimación (IC 95\%) } \\
\hline 3,000 & $(2,717$ & $3,283)$ \\
\hline 4,500 & $(4,217$ & $4,783)$ \\
\hline 9,500 & $(9,217$ & $9,783)$ \\
\hline 5,667 & $(1,815$ & $9,518)$ \\
\hline$-35,000$ & $(-35,237$ & $-34,763)$ \\
\hline 15,000 & $(14,791$ & $15,209)$ \\
\hline$-10,000$ & $(-58,999$; & $38,999)$ \\
\hline$-15,000$ & $(-15,265$ & $-14,735)$ \\
\hline$-25,000$ & $(-25,265$ & $-24,735)$ \\
\hline$-35,000$ & $(-35,265$ & $-34,735)$ \\
\hline$-65,000$ & $(-65,265$ & $-64,735)$ \\
\hline$-35,000$ & $(-56,170$ & $-13,830)$ \\
\hline$-21,000$ & $(-2,003$ & $-20,997)$ \\
\hline$-23,000$ & $(-23,003$ & $-22,997)$ \\
\hline$-2,000$ & $(-2,003$ & $-1,997)$ \\
\hline 4,000 & $(3,997$ & $4,003)$ \\
\hline$-10,500$ & $(-23,758$ & $2,758)$ \\
\hline$-34,000$ & $(-39,276$ & $-28,724)$ \\
\hline$-34,000$ & $(-39,276$ & $-28,724)$ \\
\hline$-3,500$ & $(-3,560$ & $-3,440)$ \\
\hline$-3,500$ & $(-3,560$ & $-3,440)$ \\
\hline$-7,000$ & $(-7,101$ & $-6,899)$ \\
\hline$-12,500$ & $(-12,601$ & $-12,399)$ \\
\hline$-17,500$ & $(-17,601$ & $-17,399)$ \\
\hline$-12,333$ & $(-18,276$ & $-6,390)$ \\
\hline$-9,000$ & $(-9,007$ & $-8,993)$ \\
\hline$-9,000$ & $(-9,007$ & $-8,993)$ \\
\hline 18,500 & $(18,380$ & $18,620)$ \\
\hline 19,000 & $(18,880$ & $19,120)$ \\
\hline 18,000 & $(17,880$ & $18,120)$ \\
\hline 18,500 & $(17,934$ & $19,066)$ \\
\hline$-19,000$ & $(-19,310$ & $-18,690)$ \\
\hline 34,000 & $(33,690$ & $34,310)$ \\
\hline 7,500 & $(-44,439$ & $59,439)$ \\
\hline 2,000 & $(1,987$ & $2,013)$ \\
\hline 2,000 & $(1,987$ & $2,013)$ \\
\hline 14,000 & $(13,994$ & $14,006)$ \\
\hline 14,000 & $(13,994 ;$ & $14,006)$ \\
\hline$-6,964$ & $(-12,421$ & $-1,507)$ \\
\hline
\end{tabular}
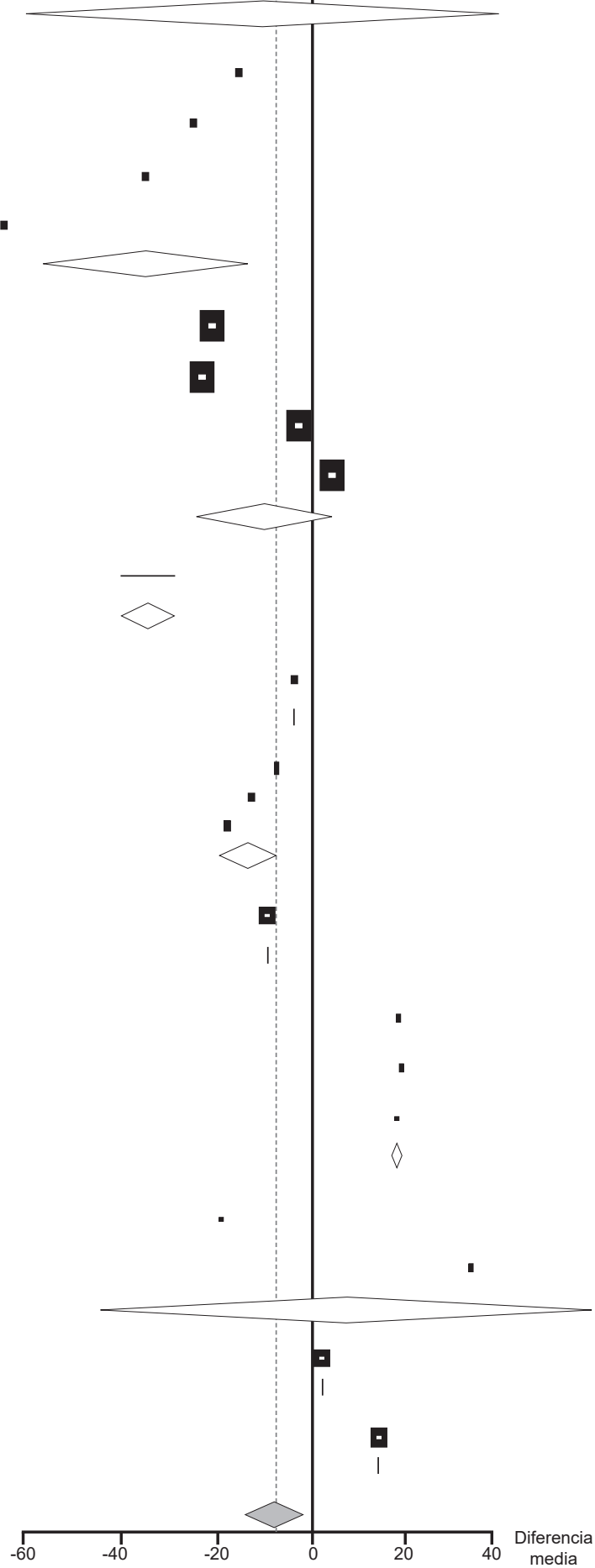

Figura 5. Intervenciones ajustadas por tamaño de muestra. 


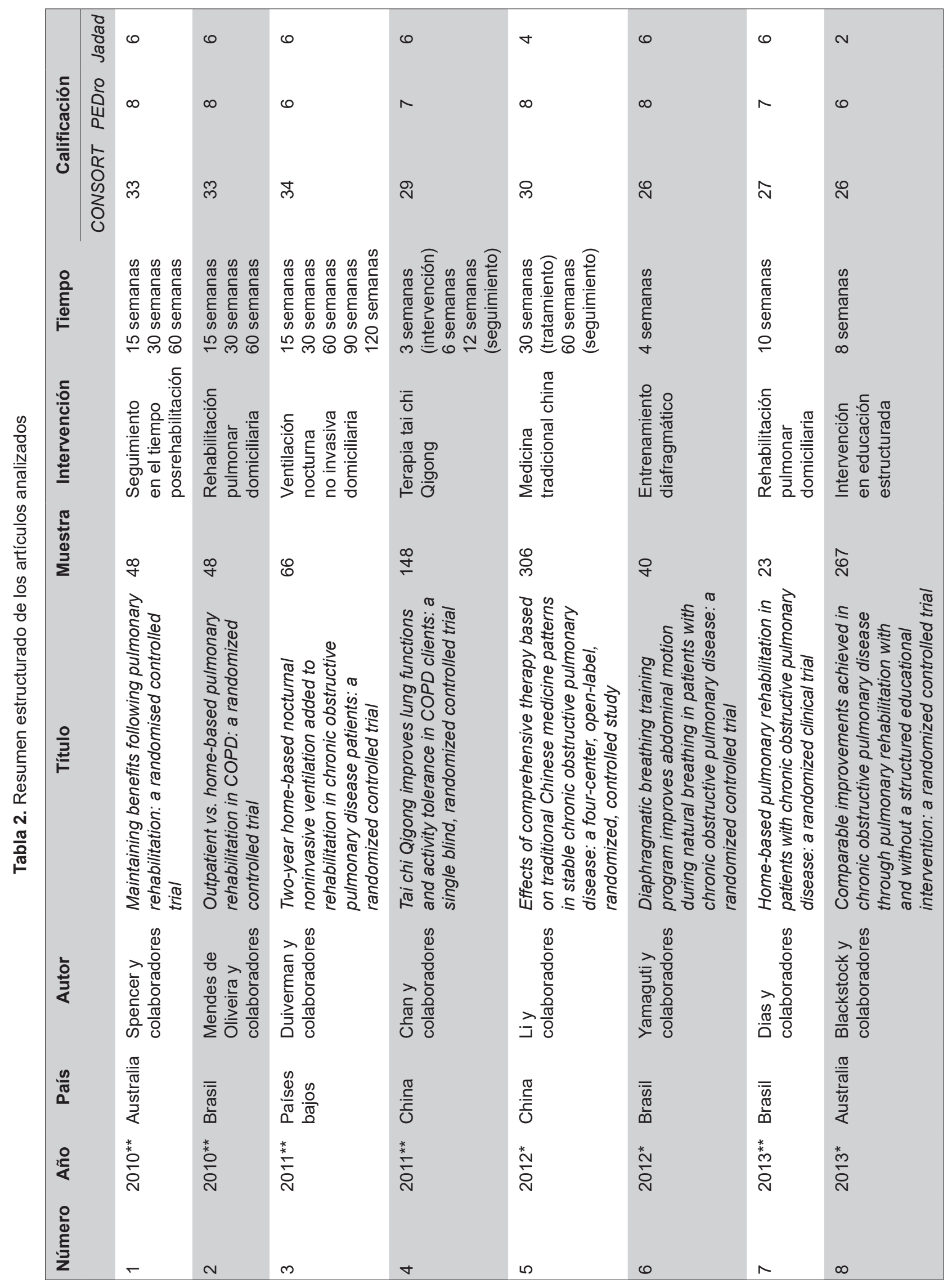




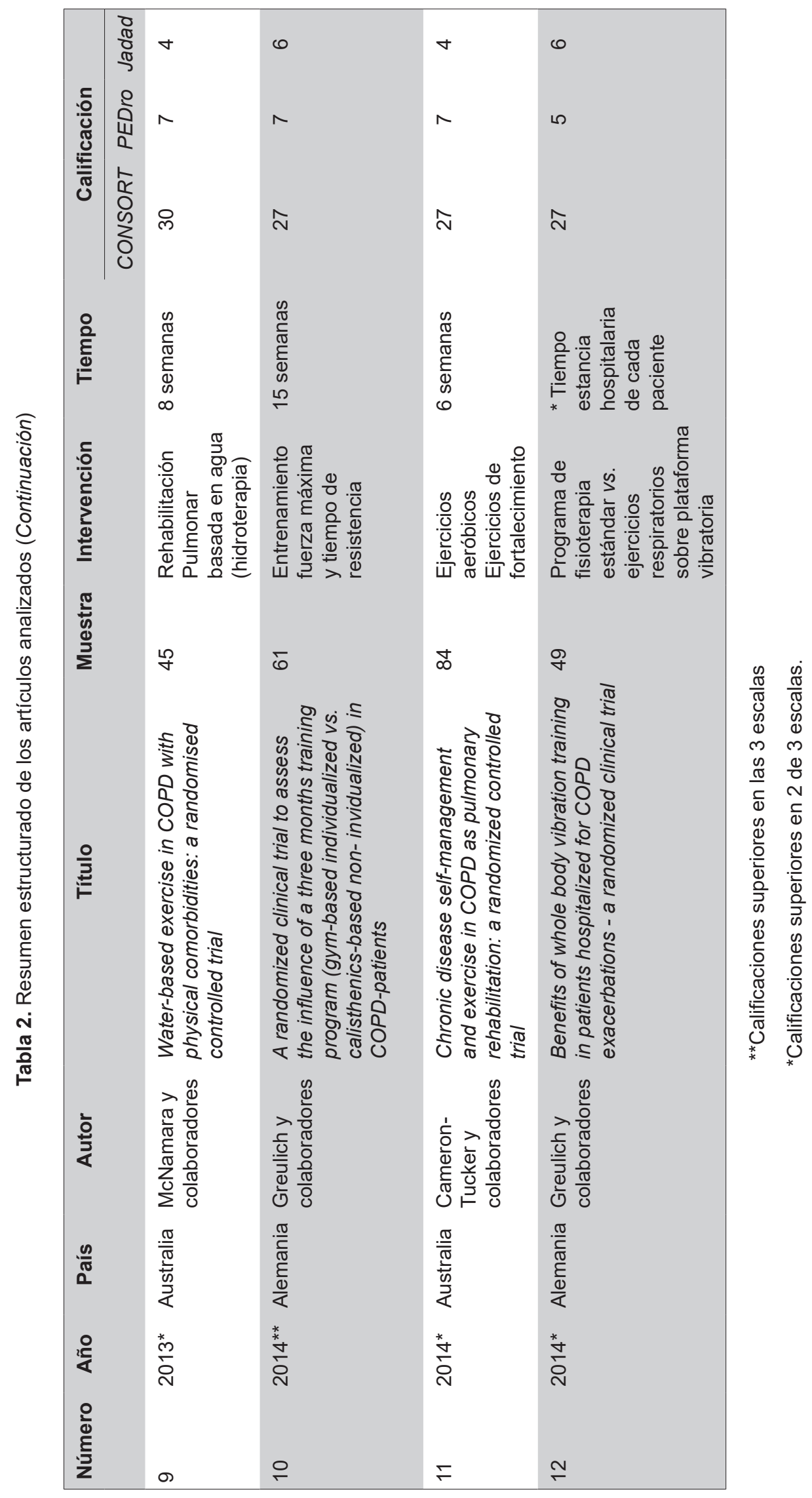


tes con enfermedad pulmonar obstructiva crónica (40). Ambos hallazgos fueron concordantes con lo evidenciado en los resultados de la presente investigación. Una segunda publicación, tipo revisión sistemática de la literatura, determinó el papel benéfico de la terapia herbal tradicional china en el manejo de los pacientes con EPOC estable (41). Empero, en las dos publicaciones los autores indicaron la interpretación cauta de los resultados debido a las limitaciones metodológicas en los estudios base; en especial, en lo concerniente al cegamiento, limitación encontrada en el análisis de los actuales resultados. Los patrones de resultados antes citados fueron repetitivos en distintas intervenciones: rehabilitación domiciliaria, ventilación mecánica no invasiva, soporte nutricional, entre otras (42-44).

En un ensayo multicéntrico realizado por Puhan y colaboradores se resaltó la importancia de la rehabilitación pulmonar para la mejoría, en especial, de la calidad de vida (45). No obstante, los autores indicaron que no se evidenció reducción en la cantidad ni el grado de exacerbaciones cuando los pacientes presentaron EPOC grave. Situación que difiere en pacientes con EPOC leve o moderada, ya que resalta una significativa disminución de la disnea, resultados que se asemejan a lo indicado por la mayoría de las publicaciones analizadas. Frente a este elemento, una revisión sistemática realizada por Jácome y colaboradores analizó el papel de la rehabilitación pulmonar en pacientes con EPOC leve, encontrando mejoría en los desenlaces evaluados. No obstante, se sugirieron nuevos estudios dado que dicha revisión solo contó con 3 artículos base con diseños metodológicos distintos.

De acuerdo con los resultados obtenidos, se considera que existe una marcada heterogeneidad en las publicaciones analizadas, situación que confirma lo planteado en la hipótesis del artículo. Al respecto, en una revisión sistemática de la literatura sobre EPOC y rehabilitación pulmonar publicada por McCarthy, con la colaboración Cochrane, se resaltaron las múltiples limitaciones con el cálculo de la muestra y cegamiento de las intervenciones, ante lo cual, se argumentó que se debe, en parte, a la naturaleza de los estudios evaluados, hallazgos concordantes con lo encontrado de forma repetitiva a través de la estrategia CONSORT y la escala PEDro, donde además se encontró que solo el $19,57 \%$ de las publicaciones analizadas contaba con acceso al protocolo de trabajo completo, lo que en la mayoría de los casos significó un punto crítico en la calidad metodológica de las publicaciones (39).

En una revisión de metaanálisis previo con un estudio realizado por Pan y colaboradores se confirmó la marcada heterogeneidad en los tratamientos, no solo en rehabilitación, sino en el abordaje desde la medicina tradicional, lo que serviría de punto de partida para nuevos estudios (46). Los autores también resaltaron la pobre prevención de la enfermedad y las intervenciones desde la rehabilitación en estadios avanzados, a lo que también se le puede atribuir la diferencia en los resultados obtenidos.

En la mayoría de los estudios se recalca la heterogeneidad estadísticamente significativa como un punto crítico en el abordaje de este tema, así lo menciona Li en su metaanálisis realizado sobre la alteración de gen y polimorfismos asociados con EPOC.

Hasta el momento y de acuerdo con el conocimiento de los autores, una de las fortalezas de la presente revisión es que es la primera publicación realizada con el objetivo de valorar publicaciones de pacientes con fenotipo mixto sometidos a rehabilitación pulmonar; además, se contó con un total de 12 publicaciones de alta calidad para el desarrollo del metaanálisis. Se presentaron limitaciones importantes que se deben considerar al momento de establecer generalizaciones: la primera fue que se realizó una búsqueda con el filtro de publicaciones solo en español o inglés, dejando por fuera otros idiomas, lo que corresponde a un sesgo de selección; un segundo factor limitante fue la exclusión de búsqueda de literatura gris. Frente al uso de escalas de valoración, se encontró que las escalas CONSORT y PEDro no son aptas para la calificación de estudios aleatorizados. Al respecto, se consideró utilizar estas escalas dado su consistencia metodológica, complementando la evaluación con el uso de la escala Jadad, que compensó la limitación implicada. Los elementos antes expuestos se deben tener en cuenta en futuras publicaciones.

\section{Conclusiones}

Luego de la realización de la presente revisión sistemática, se concluye que se necesitan nuevas inves- 
tigaciones metodológicamente óptimas que valoren procesos de rehabilitación pulmonar en pacientes con diagnóstico de fenotipo mixto o EPOC, nacional e internacionalmente..

\section{Conflicto de interés}

Los autores no declaran conflictos de interés y no se recibió patrocinio de ninguna empresa farmacéutica al momento del desarrollo de la investigación.

\section{Referencias}

1. Martínez C, González F, Ramírez J, González I, Alfageme I, Orejas $\mathrm{C}$, et al. Recomendaciones para la evaluación médica de la capacidad laboral en el enfermo respiratorio crónico. Arch Bronconeumol. 2013;49(11):480-90. DOI: 10.1016/j. arbres.2013.06.004.

2. Asociación Latinoamericana del Tórax (ALAT). Proyecto latinoamericano de investigación en obstrucción pulmonar. 2006. Disponible en: http://www.platino-alat.org/docs/libro_platino_es.pdf

3. Buist AS, McBurnie MA, Vollmer WM, Gillespie S, Burney P, Mannino DM, et al. International variation in the prevalence of COPD (The BOLD study): a population-based prevalence study. Lancet. 2007;370(9589):741-50. DOI: 10.1016/S01406736(07)61377-4.

4. Buist AS, Vollmer WM, McBurnie MA. Worldwide burden of COPD in high- and low-income countries. Part I. The burden of obstructive lung disease (BOLD) initiative. Int J Tuberc Lung Dis. 2008;12(7):703-8.

5. Global Initiative For Chronic Obstructive Lung Disease. Global Strategy for the Diagnosis, Management, and Prevention of Chronic Obstructive Pulmonary Disease (update 2016). GOLD; 2016. Disponible en: www.goldcopd.org.

6. Organización Mundial de la Salud. Enfermedades respiratorias crónicas. Disponible en: http://www.who.int/respiratory/ asthma/es/

7. World Health Organization. World health statistics 2014. 2014. Disponible en: http://apps.who.int/iris/bitstre am/10665/112738/1/9789240692671_eng.pdf

8. Rennard SI, Vestbo J. The many "small COPDs": COPD should be an orphan disease. Chest. 2008;134(3):623-7. DOI: 10.1378/chest.07-3059.

9. Vestbo J, Anderson W, Coxson HO, Crim C, Dawber F, Edwards L, et al. Evaluation of COPD longitudinally to identify predictive surrogate end-points (ECLIPSE). Eur Respir J. 2008;31:869-73. DOI: 10.1183/09031936.00111707.

10. García-Aymerich J, Agustí A, Barberà JA, Belda J, Farrero E, Ferrer A, et al. La heterogeneidad fenotípica de la EPOC.
Arch Bronconeumol. 2009;45:129-38. DOI: 10.1016/j.arbres.2008.10.001

11. Postma DS, Kerkhof M, Boezen HM, Koppelman GH. Asthma and chronic obstructive pulmonary disease. Common genes, common environments? Am J Respir Crit Care Med. 2011;183(12):1588-94. DOI: 10.1164/rccm.201011-1796PP.

12. Miravitlles M, Soler-Cataluña JJ, Calle M, Molina J, Almagro P, Quintano J, et al. Guía española de la EPOC (GesEPOC). Arch Bronconeumol. 2014;50(1):1-16. DOI: 10.1016/S03002896(14)70070-5.

13. Hurst JR, Vestbo J, Anzueto A, Locantore N, Mullerova $H$, Tal-Singer $R$, et al. Susceptibility to exacerbation in chronic obstructive pulmonary disease. $\mathrm{N}$ Engl $\mathrm{J}$ Med. 2010;363(12):1128-38. DOI: 10.1056/NEJMoa0909883.

14. Sobradillo P, García-Aymerich J, Agustí A. Fenotipos clínicos de la EPOC. Arch Bronconeumol. 2010;46(11):8-11. DOI: 10.1016/S0300-2896(10)70056-9.

15. Agustí AG. COPD, a multicomponent disease: implications for management. Respir Med. 2005;99(6):670-82. DOI: 10.1016/j.rmed.2004.11.006.

16. Gibson PG, Simpson JL. The overlap syndrome of asthma and COPD: what are its features and how important is it? Thorax. 2009;64(8):728-35. DOI: 10.1136/thx.2008.108027.

17. Anderson GP. Endotyping asthma: new insights into key pathogenic mechanisms in a complex, heterogeneous disease. Lancet. 2008;372(9643):1107-19. DOI: 10.1016/S01406736(08)61452-X.

18. Mets OM, Murphy K, Zanen P, Gietema HA, Lammers JW, van Ginneken B, et al. The relationship between lung function impairment and quantitative computed tomography in chronic obstructive pulmonary disease. Eur Radiol. 2012;22(1):120-8. DOI: $10.1007 / \mathrm{s} 00330-011-2237-9$.

19. Karadag F, Karul AB, Cildag O, Altun C, Gurgey O. Determinants of BMI in patients with COPD. Respirology. 2004:9(1):70-5. DOI: 10.1111/j.1440-1843.2003.00533.x.

20. Yang L, Zhou M, Smith M, Yang G, Peto R, Wang J, et al. Body mass index and chronic obstructive pulmonary diseaserelated mortality: a nationally representative prospective study of 220000 men in China. Int J Epidemiol. 2010;39(4):102736. DOI:10.1093/ije/dyq051.

21. Cobos-Carbó A, Augustosvky F. Declaración CONSORT 2010: actualización de la lista de comprobación para informar ensayos clínicos aleatorizados de grupos paralelos. Med Clin (Barc). 2011;137(5):213-5. DOI: 10.1016/j.medcli.2010.09.034.

22. Moher D, Jadad AR, Nichol G, Penman M, Tugwell P, Walsh S. Assessing the quality of randomized controlled trials: an annotated bibliography of scales and checklists. Control Clin Trials. 1995;16(1):62-73. DOI: 10.1016/01972456(94)00031-W. 
23. Moseley AM, Herbert RD, Sherrington C, Maher CG. Evidence for physiotherapy practice: a survey of the Physiotherapy Evidence Database (PEDro). Aust J Physiother. 2002;48(1):43-9. DOI: 10.1016/S0004-9514(14)60281-6.

24. Spencer LM, Alison JA, McKeough ZJ. Maintaining benefits following pulmonary rehabilitation: a randomised controlled trial. Eur Respir J. 2010;35(3):571-7. DOI: 10.1183/09031936.00073609.

25. Mendes de Oliveira J, Leitão Filho F, Malosa Sampaio L, Negrinho de Oliveira A, Pastrello Hirata R, Costa D, et al. Outpatient vs. home-based pulmonary rehabilitation in COPD: a randomized controlled trial. Multidiscip Respir Med. 2010;5(6):401-8. DOI: 10.1186/2049-6958-5-6-401.

26. Duiverman M, Wempe J, Bladder G, Vonk J, Zijlstra J, Kerstjens $\mathrm{H}$, et al. Two-year home-based nocturnal noninvasive ventilation added to rehabilitation in chronic obstructive pulmonary disease patients: a randomized controlled trial. Respir Res. 2011;12:112. DOI: https://doi.org/10.1186/1465-9921$12-112$.

27. Chan AW, Lee A, Suen LK, Tam WW. Tai chi Qigong improves lung functions and activity tolerance in COPD clients: a single blind, randomized controlled trial. Complement Ther Med. 2011;19(1):3-11. DOI: 10.1016/j.ctim.2010.12.007.

28. Dias F, Sampaio L, da Silva G, Gomes E, do Nascimento E, Alves V, et al. Home-based pulmonary rehabilitation in patients with chronic obstructive pulmonary disease: a randomized clinical trial. Int J Chron Obstruct Pulmon Dis. 2013;8:537-54. DOI: 10.2147/COPD.S50213.

29. Greulich T, Kehr K, Nell C, Koepke J, Haid D, Koehler U, et al. A randomized clinical trial to assess the influence of a three months training program (gym-based individualized vs. calisthenics-based non-invidualized) in COPD-patients. Resp Res. 2014;15(1):36. DOI: https://doi.org/10.1186/1465-992115-36.

30. Li S, Li J, Wang M, Xie Y, Yu X, Sun Z, et al. Effects of comprehensive therapy based on traditional Chinese medicine patterns in stable chronic obstructive pulmonary disease: a four-center, open-label, randomized, controlled study. BMC Complement Altern Med. 2012;12:197. DOI: 10.1186/14726882-12-197.

31. Yamaguti WP, Claudino RC, Neto AP, Chammas MC, Gomes AC, Salge JM, et al. Diaphragmatic breathing training program improves abdominal motion during natural breathing in patients with chronic obstructive pulmonary disease: a randomized controlled trial. Arch Phys Med Rehabil. 2012;93(4):571-7. DOI: 10.1016/j.apmr.2011.11.026.

32. Blackstock FC, Webster KE, McDonald CF, Hill CJ. Comparable improvements achieved in chronic obstructive pulmonary disease through pulmonary rehabilitation with and without a structured educational intervention: a randomized controlled trial. Respirology. 2014;19(2):193-202. DOI: 10.1111/resp. 12203 .
33. McNamara RJ, McKeough ZJ, McKenzie DK, Alison JA. Water-based exercise in COPD with physical comorbidities: a randomized controlled trial. Eur Respir J. 2013;41(6):128491. DOI: 10.1183/09031936.00034312.

34. Cameron-Tucker HL, Wood-Baker R, Owen C, Joseph L, Walters EH. Chronic disease self-management and exercise in COPD as pulmonary rehabilitation: a randomized controlled trial. Int J Chron Obstruct Pulmon Dis. 2014;9:513-23. DOI: 10.2147/COPD.S58478.

35. Greulich T, Nell C, Koepke J, Fechtel J, Franke M, Schmeck $\mathrm{B}$, et al. Benefits of whole body vibration training in patients hospitalised for COPD exacerbations - a randomized clinical trial. BMC Pulm Med. 2014;14:60. DOI: 10.1186/1471-246614-60.

36. Fu JJ, Gibson PG, Simpson JL, McDonald VM. Longitudinal changes in clinical outcomes in older patients with asthma, COPD and asthma-COPD overlap syndrome. Respiration. 2014;87(1):63-74. DOI: 10.1159/000352053.

37. Postma DS, Rabe KF. The asthma-COPD overlap syndrome. N Engl J Med. 2015;373(13):1241-9. DOI: 10.1056/NEJMra1411863.

38. COPD Working Group. Pulmonary rehabilitation for patients with chronic pulmonary disease (COPD): an evidence-based analysis. Ont Health Technol Assess Ser. 2012;12(6):1-75.

39. McCarthy B, Casey D, Devane D, Murphy K, Murphy E, Lacasse Y. Pulmonary rehabilitation for chronic obstructive pulmonary disease. Cochrane Database Syst Rev. 2015;23;(2):CD003793. DOI: 10.1002/14651858.CD003793.pub3.

40. Wu W, Liu X, Wang L, Wang Z, Hu J, Yan J. Effects of Tai Chi on exercise capacity and health-related quality of life in patients with chronic obstructive pulmonary disease: a systematic review and meta-analysis. Int J Chron Obstruct Pulmon Dis. 2014;9:1253-63. DOI: 10.2147/COPD.S70862.

41. An X, Zhang A, May B, Lin L, Xu L, Xue C. Oral Chinese herbal medicine for improvement of quality of life in patients with stable chronic obstructive pulmonary disease: a systematic review. J Altern Complement Med. 2012;18(8):731-43. DOI: $10.1089 / \mathrm{acm} .2011 .0389$.

42. Neves LF, Reis MH, Plentz RD, Matte DL, Coronel CC, Sbruzzi G. Expiratory and expiratory plus inspiratory muscle training improves respiratory muscle strength in subjects with COPD: systematic review. Respir Care. 2014;59(9):1381-8. DOI: $10.4187 /$ respcare.02793.

43. Ricci C, Terzoni S, Gaeta M, Sorgente A, Destrebecq A, Gigliotti F. Physical training and noninvasive ventilation in COPD patients: a meta-analysis. Respir Care. 2014;59(5):70917. DOI: $10.4187 /$ respcare.02626.

44. Collins PF, Elia M, Stratton RJ. Nutritional support and functional capacity in chronic obstructive pulmonary disease: a systematic review and meta-analysis. Respirology. 2013;18(4):616-29. DOI: 10.1111/resp.12070. 
45. Puhan MA, Spaar A, Frey M, Turk A, Brändli O, Ritscher D, et al. Early versus late pulmonary rehabilitation in chronic obstructive pulmonary disease patients with acute exacerbations: a randomized trial. Respiration. 2012;83(6):499-506 DOI: $10.1159 / 000329884$.
46. Pan L, Wang M, Xie X, Du C, Guo Y. Effects of anabolic steroids on chronic obstructive pulmonary disease: a meta-analysis of randomised controlled trials. PLoS One. 2014;9(1): e84855. DOI: 10.1371/journal.pone.0084855. 